\title{
Potential problems of stability and convergence in image-based and position-based visual servoing
}

\author{
François Chaumette \\ IRISA / INRIA Rennes \\ Campus de Beaulieu \\ 35042 Rennes cedex, France
}

\begin{abstract}
Visual servoing, using image-based control or positionbased control, generally gives satisfactory results. However, in some cases, convergence and stability problems may occur. The aim of this paper is to emphasize these problems by considering an eye-in-hand system and a positioning task with respect to a static target which constrains the six camera degrees of freedom.
\end{abstract}

\section{Introduction}

The two classical approaches of visual servoing (that is image-based control and position-based control) are different in the nature of the inputs used in their respective control schemes $[28,10,14]$. Even if the resulting robot behaviors thus also differ, both approaches generally give satisfactory results: the convergence to the desired position is reached, and, thanks to the closed-loop used in the control scheme, the system is stable, and robust with respect to camera calibration errors, robot calibration errors, and image measurements errors. That is particularly true when only few degrees of freedom are vision-based controlled (such as camera pan and tilt for target tracking), However, in some cases, convergence and stability problems may occur. The aim of this paper is to emphasize these problems with simple and concrete examples. We consider in this paper an eye-in-hand system, and a generic positioning task with respect to a static target which constrains the six camera degrees of freedom. However, most of the given results can be generalized to other kind of systems, such as for example monocular or binocular vision systems simultaneously observing a target and a robot end-effector $[1,9]$.

\section{Image-based visual servoing}

Image-based visual servoing is based on the selection in the image of a set $s$ of visual features that has to reach a desired value $s^{*}$. Usually, $s$ is composed of the image coordinates of several points belonging to the considered target. However, it may be interesting to use other geometrical visual features (such as the parameters which represent the image of a straight line, or of a sphere, etc.) in function of the vision-based task that has to be achieved and the nature 
of the present objects [6,3]. As for $s^{*}$, it is obtained either during an off-line learning step (where the robot is moved at its desired position with respect to the target and the corresponding image is acquired), either by computing the projection in the image of a $3 \mathrm{D}$ model of the target for the desired camera pose (which necessitates a perfect camera calibration and a perfect knowledge of the 3D target model in order that the camera does reach the given pose).

It is well known that the image Jacobian $J_{s}$, also called interaction matrix related to $s$, plays a crucial role in the design of the possible control laws. $J_{s}$ is defined from:

$$
\dot{s}=\left(\frac{\partial s}{\partial r}\right) \frac{d r}{d t}=J_{s} T
$$

where $T=\frac{d r}{d t}=\left(V^{T}, \Omega^{T}\right)^{T}$ is the camera velocity screw ( $V$ and $\Omega$ represent its translational and rotational component respectively). Using a classical perspective projection model with unit focal length, and if $X$ and $Y$ coordinates of image points are selected in $s, J_{s}$ is directly obtained from:

$$
\left(\begin{array}{c}
\dot{X} \\
\dot{Y}
\end{array}\right)=\left(\begin{array}{cccccc}
-1 / z & 0 & X / z & X Y & -\left(1+X^{2}\right) & Y \\
0 & -1 / z & Y / z & 1+Y^{2} & -X Y & -X
\end{array}\right) T
$$

where $z$ is the depth of the corresponding point in the camera frame.

All the existing control schemes compute the camera velocity sent to the robot controller (or directly the robot joints velocity, by introducing the robot Jacobian, if joint limits and kinematics singularities avoidance is needed $[21,17])$ with the following forms $([8,11,23]$, etc.):

$$
T=\widehat{J_{s}^{+}} \dot{s} \quad \text { or } \quad T=f\left(\widehat{J_{s}^{+}}\left(s-s^{*}\right)\right)
$$

where function $f$ may be as simple as a proportional gain [6], or a more complex function used to regulate $s$ to $s^{*}$ (optimal control [23], non-linear control [11], etc.), and $\widehat{J_{s}^{+}}$is a model, an approximation, or an estimation of the pseudoinverse of $J_{s}$. Indeed, camera calibration errors, noisy image measurements, and unknown depth $z$ involved in (2) imply the use of such model, since the real value of $J_{s}$ remains unknown.

A well known sufficient condition to ensure the global asymptotic stability of the system (that is of course non-linear since $X$ and $Y$ are involved in (2)) is [24]:

$$
\widehat{J_{s}^{+}} J_{s}(s(t), z(t))>0, \forall t
$$

This condition, even if it is difficult to exploit in practice, allows one to set the possible choices for $\widehat{J_{s}^{+}}$. In fact, three different cases have been considered in the literature:

- $J_{s}^{+}=J_{s}^{+}(t)$. In that case, the image Jacobian is numerically estimated during the camera motion without taking into account the analytical form given by $(2)[13,15]$ (neural networks have also sometimes been used [27]). This approach seems to be very interesting if any camera and robot models are available. However, it is impossible in that case to demonstrate when condition (4) 
is ensured. Furthermore, initial coarse estimation of the image Jacobian may lead to unstable results, especially at the beginning of the servoing, and some visual features may get out of the camera field of view (see Figure 1.a).

- $\widehat{J_{s}^{+}}=J_{s}^{+}(s(t), \widehat{z}(t))$. The image Jacobian is now updated at each iteration of the control law using in (2) the current measure of the visual features and an estimation $\widehat{z}(t)$ of the depth of each considered point. $\widehat{z}(t)$ can be obtained either from the knowledge of a 3D model of the object [4], either from the measure of the camera motion [26,2]. This case seems to be optimal since, ideally, we thus have $\widehat{J_{s}^{+}} J_{s}=I I, \forall t$, which of course satisfies condition (4) and implies a perfect decoupled system. Each image point is constrained to reach its desired position following a straight line (see Figure 1.b). However, we will see that such a control in the image may imply inadequate camera motion, leading to possible local minima and the nearing of task singularities.

- $\widehat{J_{s}^{+}}=J_{s}^{+}\left(s^{*}, \widehat{z^{*}}\right)$. In this last case, $\widehat{J_{s}^{+}}$is constant and determined during an off-line step using the desired value of the visual features and an approximation of the points depth at the desired camera pose. Condition (4) is now ensured only in a neighborhood of the desired position, and a decoupled behavior will be achieved only in a smaller neighborhood. Determining analytically the limits of these neighborhoods seems to be out of reach owing to the complexity of the involved symbolic computations. The performed trajectory in the image may be quite unforeseeable, and some visual features may get out of the camera field of view during the servoing (leading of course to its failure), especially if the initial camera position is far away from its desired one (see Figure 1.c).

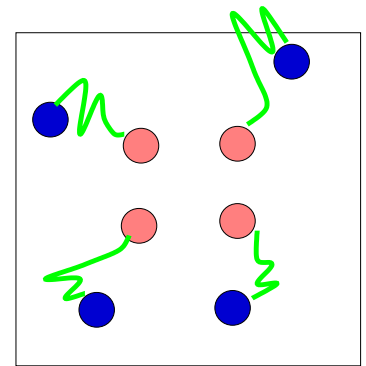

a) $\widehat{J_{s}^{+}}(t)$

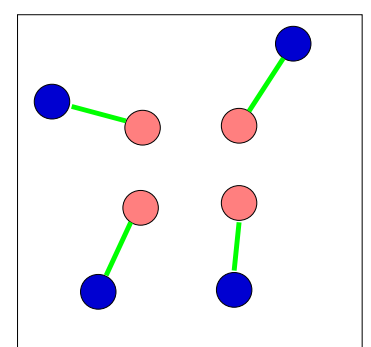

b) $J_{s}^{+}(s(t), \widehat{z}(t))$

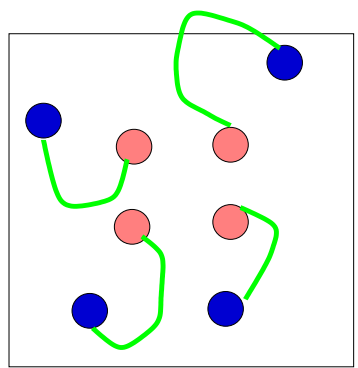

c) $J_{s}^{+}\left(s^{*}, \widehat{z^{*}}\right)$

Fig. 1. Possible choices for $\widehat{J_{s}^{+}}$and corresponding behavior: black points represent the initial position of the target in the image, and gray points and lines respectively represent its desired position and a possible trajectory in the image)

Image-based visual servoing is known to be generally satisfactory, even in the presence of important camera or hand-eye calibration errors [5]. However, we now exhibit the following stability and convergence problems which may occur: 
- $\widehat{J}$, or even $J$, may become singular during the servoing, which of course leads to an unstable behavior.

- local minima may be reached owing to the existence of unrealizable image motions.

\subsection{Reaching or nearing a task singularity}

It is well known that the image Jacobian is singular if $s$ is composed by the image of three points such that they are collinear, or belong to a cylinder containing the camera optical center $[3,19,22]$. Using more than three points generally allows us to avoid such singularities. However, we now demonstrate by a concrete example that, whatever the number of points and their configuration, the image Jacobian may become singular during the visual servoing, if image points are chosen as visual features.

Let us consider that the camera motion from its initial to desired poses is a pure rotation of $180 \mathrm{dg}$ around the optical axis. This 3D motion leads to an image motion corresponding to a symmetry around the principal point. If $J_{s}^{+}(s(t), \widehat{z}(t))$ is used in the control scheme and perfect measurements and estimations are assumed, we can note that $\widehat{J_{s}^{+}} J_{s}=I$ for the initial camera position, which leaves us to expect a correct behavior. However, the obtained image trajectory of each point is a straight line such that all the points lie at the principal point at the same instant (see Figure 2.a). It corresponds to a pure backward translational camera motion along the optical axis (and unfortunately to a zero rotational motion around the optical axis), that moves the camera at infinity. At this unexpected position, the image Jacobian of each point $i$ is given by (see (2)):

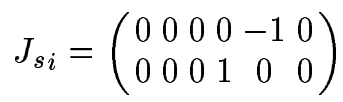

The interaction matrix $J_{s}$, and $\widehat{J_{s}^{+}}$, are here of rank 2 , instead of 6 , which of course corresponds to a task singularity, and where condition (4) is no more ensured. Let us now consider the case where $J_{s}^{+}\left(s^{*}, \widehat{z^{*}}\right)$ is used in the control scheme. This choice implies that the control law behaves as if the error in the image was as small as possible. It is clear from Figure 2.b (where white points correspond to such near position) that the obtained camera motion is now a pure forward translational motion along the optical axis (and, once again, without any rotational motion around the optical axis). The camera thus moves directly toward the target (in practice, the collision is avoided thanks to the getting out of the visual features from the image), and toward a singularity of $J_{s}$. Indeed, when $z=0$, for all points not lying on the optical center, $J_{s i}$ is given by:

$$
J_{s i} \rightarrow\left(\begin{array}{cccccc}
\infty & 0 & \infty & \infty & \infty & \infty \\
0 & \infty & \infty & \infty & \infty & \infty
\end{array}\right)
$$

It is interesting to note that, in that case, $J_{s}^{+}\left(s^{*}, \widehat{z^{*}}\right)$, which is used in the control scheme, is not singular. However, the problem occurs owing to the singularity of $J_{s}$, which is involved in condition (4). 
In the two previous cases, the reaching of the singularity can be avoided if the camera rotation is less important. However, the coupling between translational and rotational camera motion implies a really unsatisfactory camera trajectory, by the nearing (and then the moving away) of the singularity. Furthermore, even for the considered initial position, the singularity can be avoided, and a perfect camera trajectory can be achieved (that is a pure rotational camera motion around its optical axis) if straight lines are used in $s$ instead of points (see Figure 2.c). Indeed, by considering the $(\rho, \theta)$ parameters which describe the position in the image of a straight line [6], we obtain the results depicted on Figure 3. In the presented simulation results, we have used four lines from the six that can be defined from four points. As can be seen on the plots, the four errors $\rho_{i}-\rho_{i}^{*}$ always remain to zero, while the four $\theta_{i}-\theta_{i}^{*}$ simultaneously converges from $-180 \mathrm{dg}$ to $0 \mathrm{dg}$, thanks to the computation of a pure camera rotational motion around its optical axis. Let us note that similar experimental results can be obtained (calibration errors and noisy image measurements of course introduce small perturbations), and that these results do not depend on the number nor the configuration of the considered features.

Let us finally claim that we here have demonstrated that using straight lines is sometimes more interesting than using points, but nothing more. Indeed, some singularities may perhaps appear with straight lines for particular configurations, and some camera motion may perhaps be more adequately achieved using points (or anything else) than straight lines.

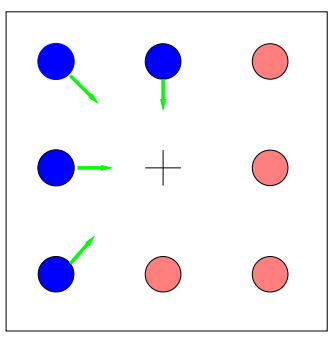

(a)

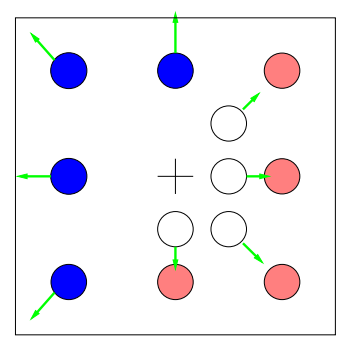

(b)

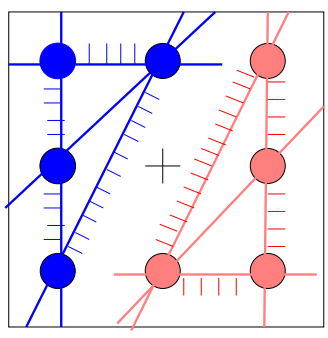

(c)

Fig. 2. Reaching (or not) a singularity: a) image motion using $\left.J_{s}^{+}(s(t), \widehat{z}(t)), \mathrm{b}\right)$ image motion using $\left.J_{s}^{+}\left(s^{*}, \widehat{z^{*}}\right), \mathrm{c}\right) s$ defined using $2 \mathrm{D}$ straight lines
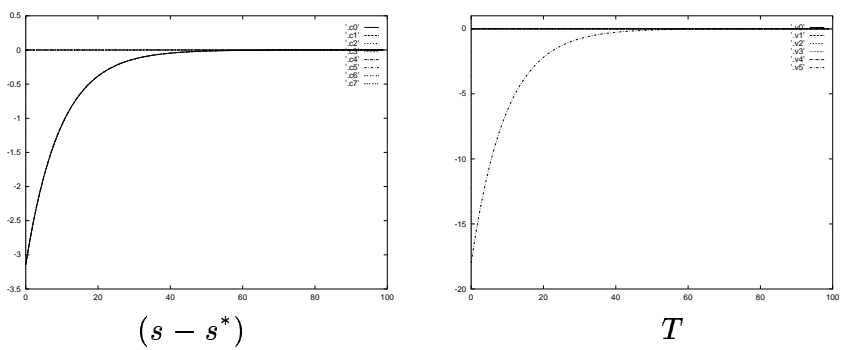

Fig. 3. Perfect behavior using 2D straight lines in $s$ 


\subsection{Reaching local minima}

We now focus on another potential problem that may appear in practice. By definition, local minima are defined such that $T=0$ and $s \neq s^{*}$ (or $\dot{s} \neq 0$ ). This is equivalent to:

$$
s-s^{*} \in \operatorname{Ker} \widehat{J_{s}^{+}} \quad\left(\text { or } \dot{s} \in \operatorname{Ker} \widehat{J_{s}^{+}}\right)
$$

If $s$ is composed by three image points (such that $J_{s}$ is full rank 6), Ker $\widehat{J_{s}^{+}}=0$, which implies that there is none local minima. However, it is well known that the same image of three points can be seen from four different camera poses [12]. In other words, there exist four camera poses (that is four global minima) such that $s=s^{*}$ (or $\dot{s}=0$ ). A unique pose can theoretically be obtained by using at least four points. However, in that case, $J_{s}$ is of dimension $8 \times 6$, which implies that $\operatorname{dim}$ Ker $\widehat{J_{s}^{+}}=2$. This does not demonstrate that local minima always exist. Indeed, the configurations $s$ such that $s-s^{*}($ or $\dot{s}) \in \operatorname{Ker} \widehat{J_{s}^{+}}$ must be physically coherent (which means that a corresponding camera pose exists). The complexity of the involved symbolic computations seems to make impossible the determination of general results. Particular cases can however be exhibited. In Figure 4 are presented the simulation results for a planar target composed of four points obtained using $J_{s}(s(t), \widehat{z}(t))$ in the control scheme. As can be seen, the visual features simultaneously decrease owing to the used strategy. However, a local minimum is reached since the camera velocity is zero while the final camera position is far away from its desired one. At that position, the error $s-s^{*}$ in the image do not completely vanish (residual error is approximately one pixel on each $X$ and $Y$ coordinate). Introducing noise in the image measurement leads to the same results, which can also be obtained in real experiments.

It is interesting to note that the global minimum is correctly reached from the same initial camera position if $J_{s}\left(s^{*}, \widehat{z^{*}}\right)$ is used in the control scheme (see Figure 5). In that case, as can be seen on $s-s^{*}$, the trajectory in the image is quite surprising, as well as the computed control law, but this behavior allows the system to avoid the local minima.

In fact, potential local minima are due to the existence of unrealizable image motions. Such motions $\dot{s}^{\perp}$ are defined by $\dot{s}^{\perp} \in$ Ker $J_{s}^{T}$. Indeed, they are such that they do not belong to the range space of $J_{s}$. In other words:

$$
\dot{s}^{\perp} \notin \operatorname{Im} J_{s} \Rightarrow \dot{s}^{\perp} \in\left(\operatorname{Im} J_{s}\right)^{\perp}=\operatorname{Ker} J_{s}^{T}
$$

Once again, if $s$ is composed of four points, $\operatorname{dim} \operatorname{Ker} J_{s}^{T}=2$. An illustration, easily obtained from (2), is presented in Figure 6 in the case of four coplanar points parallel to the image plane and forming a square.

The link between local minima and unrealizable image motions is obvious since we always have Ker $J_{s}^{T}=\operatorname{Ker} J_{s}^{+}$. Indeed, if the camera reaches a position such that $\left(s-s^{*}\right) \in \operatorname{Ker} J_{s}^{T}$, the behavior of the control law using $J_{s}(s(t), \widehat{z}(t))$ implies that $\dot{s}$ must also belong to Ker $J_{s}^{T}$. By definition, there does not exist any camera motion $T$ able to produce such imposed, but unrealizable, image 


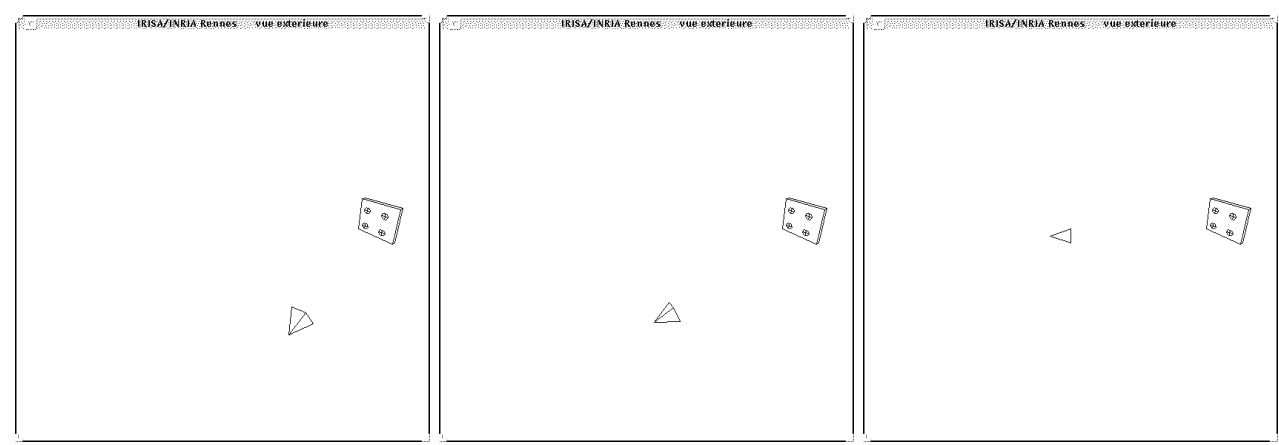

Initial pose

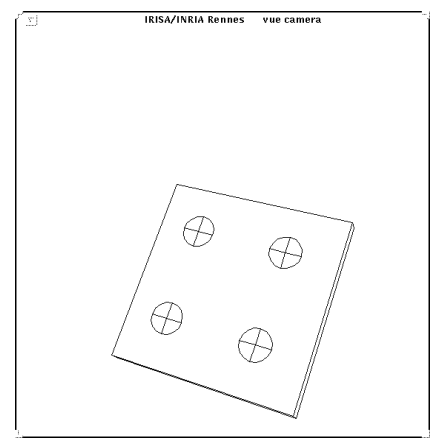

Initial image

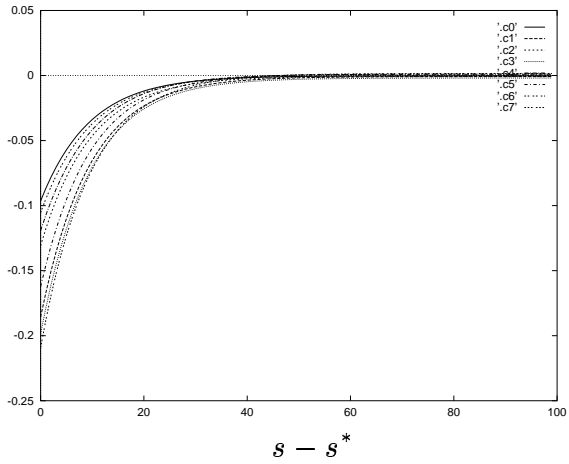

Final pose

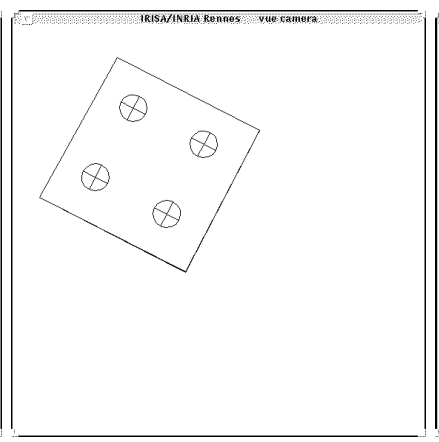

Final image
Desired pose

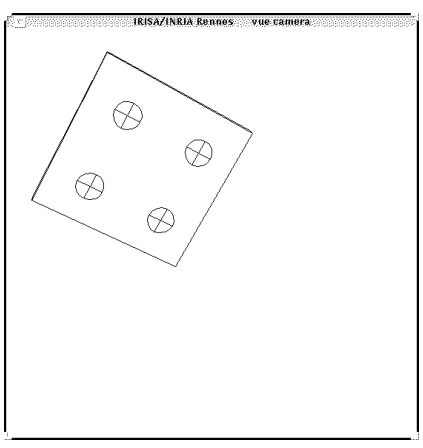

Desired image

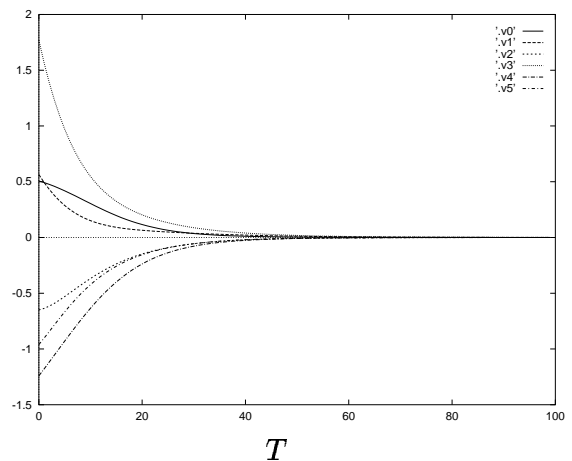

Fig. 4. Reaching a local minima using $\widehat{J_{s}}=J_{s}(s(t), \widehat{z}(t))$ 


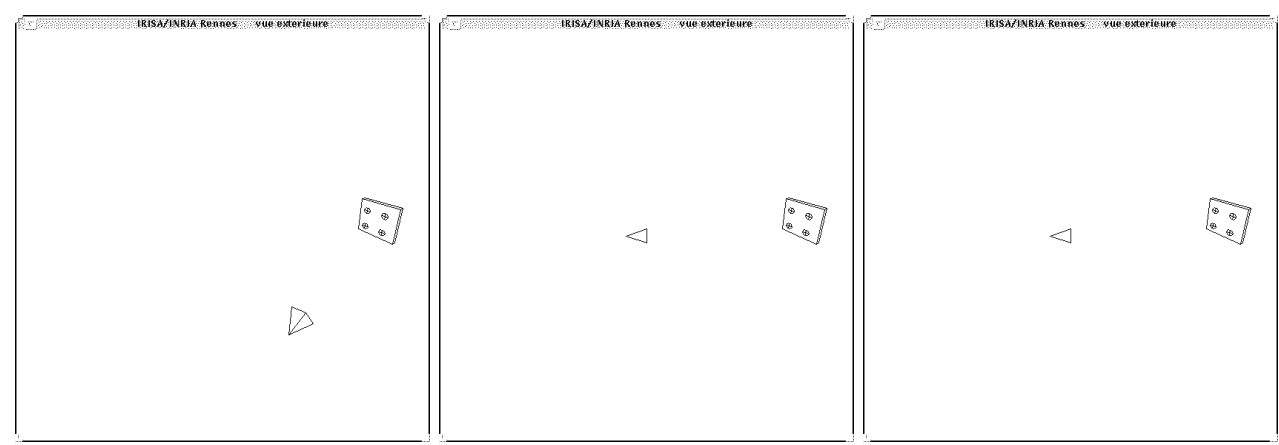

Initial pose

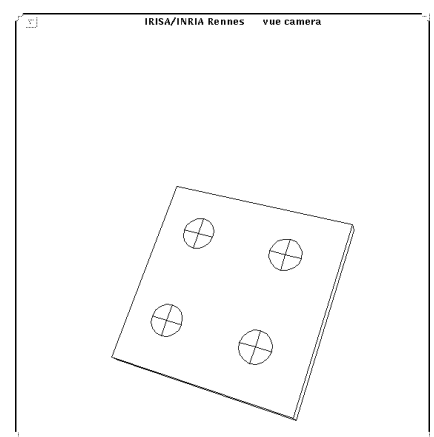

Initial image

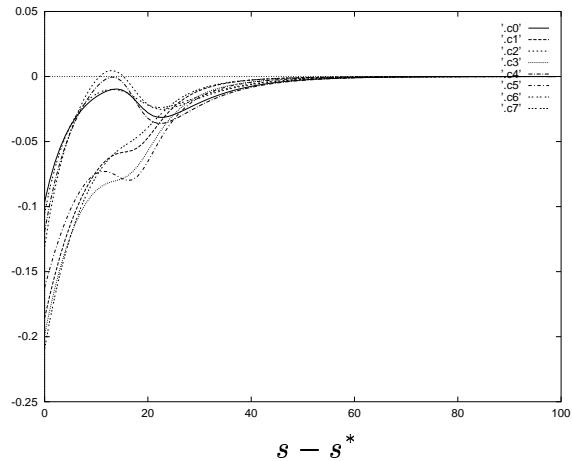

Final pose

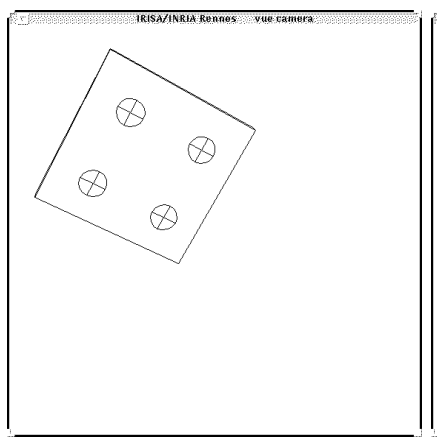

Final image
Desired pose

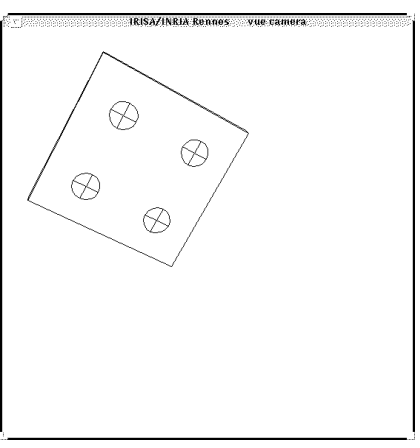

Desired image

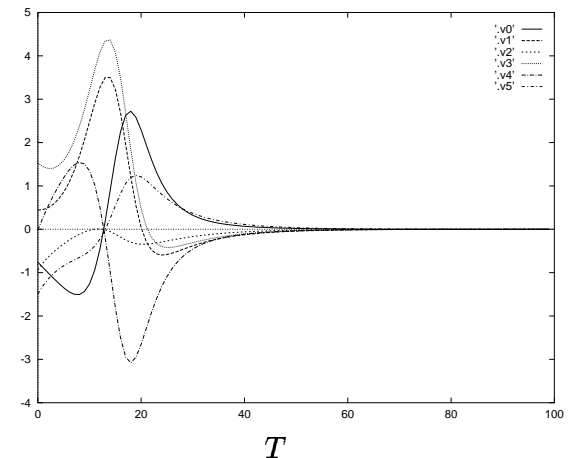

$T$

Fig. 5. Reaching the global minimum using $\widehat{J}_{s}=J_{s}\left(s^{*}, \widehat{z^{*}}\right)$ 


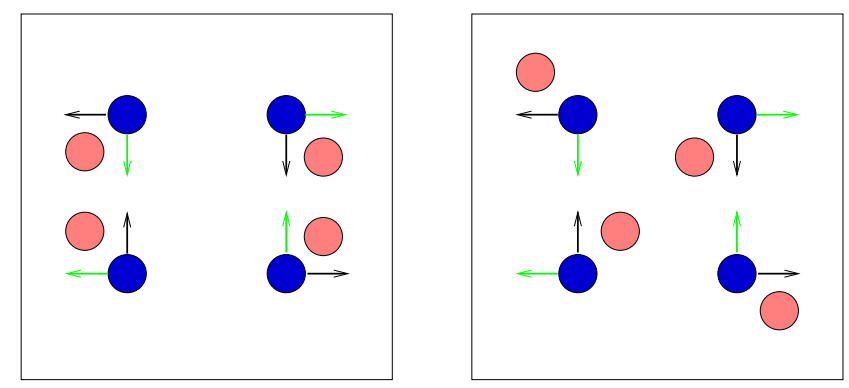

Fig. 6. Example of unrealizable image motion: arrows in gray and black respectively represent one of the two spanning vectors of $\operatorname{Ker} J_{s}^{T}$. Points in gray represent an example of image configurations unreachable from points in black using $J_{s}(s(t), \widehat{z}(t))$ in the control scheme. Reciprocally, points in black represent an example of image configurations unreachable from points in gray using $J_{s}\left(s^{*}, \widehat{z^{*}}\right)$

motions. The camera is thus in a local minima. This can be easily demonstrated since, if we assume perfect measurements and estimation, we here have $\widehat{J_{s}^{+}}=$ $J_{s}^{+}$, which of course implies that, if $\left(s-s^{*}\right) \in \operatorname{Ker} J_{s}^{T}$, then $\left(s-s^{*}\right) \in \operatorname{Ker} \widehat{J_{s}^{+}}$.

On the other hand, using $J_{s}\left(s^{*}, \widehat{z^{*}}\right)$ generally implies that $\operatorname{Ker} J_{s}^{T} \neq$ Ker $\widehat{J_{s}^{+}}$, which allows the system to avoid local minima if it computes image motions which are not unrealizable. In fact, one has to demonstrate that Ker $J_{s}^{T} \cap \operatorname{Ker} \widehat{J_{s}^{+}}=0$, or more precisely that the image configurations $\left(s-s^{*}\right)$ which belong to Ker $J_{s}^{T} \cap \operatorname{Ker} \widehat{J_{s}^{+}}$are not physically coherent. For example, it does not seem that any camera pose exists to obtain the gray image configurations depicted on Figure 6 for the considered square object.

Let us claim that we have shown in this subsection that it is sometimes more interesting to use $J_{s}\left(s^{*}, \widehat{z^{*}}\right)$ instead of $J_{s}(s(t), \widehat{z}(t))$, but nothing more. Finally, we will see in the conclusion of the paper several sufficient and simple conditions to avoid such undesirable behaviors of the image-based visual servoing.

\section{Position-based visual servoing}

Position-based visual servoing does not present the drawbacks described above. An estimation $\widehat{r}$ of the camera pose $r$ is now computed at each iteration of the control law $[12,4]$. Control schemes are thus designed in order that $\widehat{r}$ reaches a desired pose $r^{*}[29,18]$. Since the control schemes are based on inputs expressed in the Cartesian space, this approach seems to be very satisfactory. Indeed, ideally, the task Jacobian $J_{\widehat{r}}=\frac{\partial \widehat{r}}{\partial r}$ is given by:

$$
J_{\widehat{r}}=\left(\begin{array}{cc}
-I_{3} & A s(U) \\
0 & -I_{3}
\end{array}\right)
$$

where $A s(U)$ is the antisymmetric matrix associated to the vector $U$, defined by the origins of target to camera frames. Ideally, $J_{\widehat{r}}$ is thus an upper triangular 
matrix (that ensures a perfect decoupling of the camera trajectory) which is always of full rank 6 and such that Ker $J_{\widehat{r}}^{T}=\operatorname{Ker} J_{\widehat{r}}^{+}=0$. However, the form of $J_{\widehat{r}}$ given by (9) is only valid when a perfect camera calibration, perfect image measurements, a perfect $3 \mathrm{D}$ model of the object, and a perfect pose estimation algorithm are available... In fact, the real form of the task Jacobian is:

$$
J_{\widehat{r}}=\left(\frac{\partial \widehat{r}}{\partial s}\right)\left(\frac{\partial s}{\partial r}\right)
$$

The second term $\left(\frac{\partial s}{\partial r}\right)$ is nothing but the interaction matrix related to $s$ (see (1)). It is thus analytically known. On the other hand, $\left(\frac{\partial \widehat{r}}{\partial s}\right)$ which represents the variation of the estimated pose in function of a variation of the visual features, is unfortunately unknown, and depends on the used visual features, the shape of the object, the camera calibration parameters, and the used pose estimation algorithm (generally based on iterative numerical methods [4]). Theoretically demonstrating the global asymptotic stability of a position-based visual servoing seems therefore to be out of reach since a sufficient condition is given by (refer to (4)):

$$
\widehat{J_{\widehat{r}}^{+}} J_{\widehat{r}}>0, \forall t
$$

where $\widehat{J_{\widehat{r}}^{+}}$, used in the control scheme, can be chosen as (9) (it seems to be the only one possible satisfactory approximation) and where $J_{\widehat{r}}$ is, as already stated, analytically unknown.

Furthermore, since there is no more control in the image, it is impossible to ensure that the object will always remain in the camera field of view during the servoing, especially in the presence of important calibration errors. It is also well known that the Perspective from N Points problem, as most of inverse problems, is sometimes ill-posed and sensitive to perturbations (which implies a bad conditioning of $\left.\left(\frac{\partial \widehat{r}}{\partial s}\right)\right)$ : small errors in the image measurements may lead to very different results, especially in the case of a planar target, as can be seen on Figure 4 . In such cases, the control law can be completely unstable. In practice, satisfactory results are obtained if a non coplanar object is considered [29,18]. However, it would be very interesting to determine the number, the nature, and the configuration of features necessary to obtain an optimal behavior of the pose estimation

To conclude, in position-based visual servoing, most of the control problems are reported on the pose estimation algorithm. However, that does not close the whole problem of integration of vision and control.

\section{Conclusion}

As exhibited in this paper, numerous problems stand to theoretically demonstrate the complete efficiency of any visual servoing scheme. As for image-based visual servoing, an interesting challenge is to determine a universal representation of visual features adequate for any object, and from any initial camera position. It is known that the condition number of the image Jacobian plays an 
important role in the behavior of the system [7] (see also [20,25] where similar measures are presented to select the adequate visual features). More precisely, the condition number of $J_{s}$, and also of $\widehat{J_{s}^{+}}$, has to be minimal. From the results obtained in this paper, supplementary sufficient conditions to ensure a correct modeling of an image-based task are that $\operatorname{Ker} \widehat{J_{s}^{+}}=\operatorname{Ker} J_{s}^{T}=0$ in all the possible workspace (which also excludes the existence of task singularities). Intuitively, the six first inertial moments of the image of an object seem to be interesting candidates, even if observing a centered circle with such a representation (and with any unfortunately) is known to be a singular case [6]. The $2 \mathrm{D} 1 / 2$ visual servoing scheme that has been recently proposed [16], seems also to be an interesting approach to avoid convergence and stability problems.

\section{References}

1. P. Allen, A. Timcenko, B. Yoshimi, and P. Michelman. Automated tracking and grasping of a moving object with a robotic hand-eye system. IEEE Trans. on Robotics and Automation, 9(2):152-165, April 1993.

2. F. Chaumette, S. Boukir, P. Bouthemy, and D. Juvin. Structure from controlled motion. IEEE Trans. on Pattern Analysis and Machine Intelligence, 18(5):492504, May 1996.

3. F. Chaumette, P. Rives, and B. Espiau. Classification and realization of the different vision-based tasks. K. Hashimoto, editor, Visual Servoing, pages 199228, World Scientific, Singapore, 1993.

4. D. Dementhon and L. Davis. Model-based object pose in 25 lines of code. Int. Journal of Computer Vision, 15(1/2):123-141, June 1995.

5. B. Espiau. Effect of camera calibration errors on visual servoing in robotics. In Third Int. Symposium on Experimental Robotics, Kyoto, Japan, October 1993.

6. B. Espiau, F. Chaumette, and P. Rives. A new approach to visual servoing in robotics. IEEE Trans. on Robotics and Automation, 8(3):313-326, June 1992.

7. J. Feddema, C. Lee, and O. Mitchell. Automatic selection of image features for visual servoing of a robot manipulator. In IEEE Int. Conf. on Robotics and Automation, volume 2, pages 832-837, Scottsdale, Arizona, May 1989.

8. J. Feddema and O. Mitchell. Vision-guided servoing with feature-based trajectory generation. IEEE Trans. on Robotics and Automation, 5(5):691-700, October 1989.

9. G. Hager. A modular system for robust positioning using feedback from stereo vision. IEEE Trans. on Robotics and Automation, 13(4):582-595, August 1997.

10. K. Hashimoto, editor. Visual Servoing. World Scientific Series in Robotics and Automated Systems, Vol 7, World Scientific Press, Singapore, 1993.

11. K. Hashimoto and H. Kimura. Dynamic visual servoing with nonlinear modelbased control. In $12^{t h}$ World Congress IFAC, volume 9, pages 405-408, Sidney, Autralia, July 1993.

12. R. Horaud. New methods for matching $3 \mathrm{~d}$ objects with single perspective view. IEEE Trans. on Pattern Analysis and Machine Intelligence, 9(3):401-412, May 1987.

13. K. Hosoda and M. Asada. Versatile visual servoing without knowledge of true jacobian. In IEEE/RSJ Int. Conf on Intelligent Robots and Systems, pages 186193, Munchen, Germany, September 1994. 
14. S. Hutchinson, G. Hager, and P. Corke. A tutorial on visual servo control. IEEE Trans. on Robotics and Automation, 12(5):651-670, October 1996.

15. M. Jagersand, O. Fuentes, and R. Nelson. Experimental evaluation of uncalibrated visual servoing for precision manipulation. In IEEE Int. Conf. on Robotics and Automation, volume 3, pages 2874-2880, Albuquerque, New Mexico, April 1997.

16. E. Malis, F. Chaumette, and S. Boudet. Positioning a coarse-calibrated camera with respect to an unknown object by $2 \mathrm{~d} 1 / 2$ visual servoing. In IEEE Int. Conf. on Robotics and Automation, Leuven, Belgium, May 1998. (extended version available at ftp://ftp.irisa.fr/techreports/1998/PI-1166.ps.gz).

17. E. Marchand, F. Chaumette, and A. Rizzo. Using the task function approach to avoid robot joint limits and kinematic singularities in visual servoing. In IEEE/RSJ Int. Conf. on Intelligent Robots and Systems, volume 3, pages 10831090, Osaka, Japan, November 1996.

18. P. Martinet, N. Daucher, J. Gallice, and M. Dhome. Robot control using monocular pose estimation. In Workshop on New Trends in Image-based Robot Servoing, IROS'97, pages 1-12, Grenoble, France, September 1997.

19. H. Michel and P. Rives. Singularities in the determination of the situation of a robot effector from the perspective view of three points. Technical Report 1850 , INRIA, February 1993.

20. B. Nelson and P. Khosla. The resolvability ellipsoid for visual servoing. In IEEE Int. Conf. on Computer Vision and Pattern Recognition, pages 829-832, Seattle, Washington, June 1994.

21. B. Nelson and P. Khosla. Strategies for increasing the tracking region of an eyein-hand system by singularity and joint limits avoidance. Int. Journal of Robotics Research, 14(3):255-269, June 1995.

22. N. Papanikolopoulos. Selection of features and evaluation of visual measurements during robotic visual servoing tasks. Journal of Intelligent and Robotics Systems, 13:279-304, 1995.

23. N. Papanikolopoulos, P. Khosla, and T. Kanade. Visual tracking of a moving target by a camera mounted on a robot: A combination of control and vision. IEEE Trans. on Robotics and Automation, 9(1):14-35, February 1993.

24. C. Samson, M. Le Borgne, and B. Espiau. Robot Control: the Task Function Approach. Clarendon Press, Oxford, United Kingdom, 1991.

25. R. Sharma and S. Hutchinson. Optimizing hand/eye configuration for visual servo systems. In IEEE Int. Conf. on Robotics and Automation, pages 172-177, Nagoya, Japan, May 1995.

26. C. Smith and N. Papanikolopoulos. Computation of shape through controlled active exploration. In IEEE Int. Conf. on Robotics and Automation, volume 3, pages 2516-2521, San Diego, California, May 1994.

27. I. Suh. Visual servoing of robot manipulators by fuzzy membership function based neural networks. K. Hashimoto, editor, Visual Servoing, pages 285-315, World Scientific, Singapore, 1993.

28. L. Weiss, A. Sanderson, and C. Neuman. Dynamic sensor-based control of robots with visual feedback. IEEE Journal of Robotics and Automation, 3(5):404-417, October 1987.

29. W. Wilson, C. Hulls, and G. Bell. Relative end-effector control using cartesian position-based visual servoing. IEEE Trans. on Robotics and Automation, 12(5):684-696, October 1996. 